\title{
Optimal Control of a Sex-Structured HIV/AIDS Model with Condom Use
}

\author{
G. Magombedze ${ }^{1}$, Z. Mukandavire ${ }^{2}$, C. Chiyaka ${ }^{3}$ \\ and G. Musuka ${ }^{4}$ \\ ${ }^{1,2,3}$ Department of Applied Mathematics \\ ${ }^{1,2,3}$ P O Box AC939 Ascot, Bulawayo, Zimbabwe \\ E-mail(corresp.): gmagombedze@nust.ac.zw; gmagombedze@gmail.com \\ E-mail: zmukandavire@nust.ac.zw; cchiyaka@nust.ac.zw \\ ${ }^{4}$ African Comprehensive HIV/AIDS Partnerships \\ Private Bag X033 Gaborone, Botswana \\ E-mail: gnmusuka@hotmail.com
}

Received July 28, 2008; revised January 20, 2009; published online November 10, 2009

\begin{abstract}
Optimal control theory is applied to a sex-structured HIV/AIDS model with condom use as an intervention strategy. An objective functional to maximise condom use in a population and minimise cases of infectious HIV is adopted. The optimal control is characterised and solved numerically. Simulation results suggest that high percentage of condom usage is associated with reduced HIV incidence, while high costs of condom usage campaigns reduces the percentage condom usage. Targeting issuance of condoms to infectious individuals enables reduction of condom usage campaign costs, hence ensures high percentage of condom usage.
\end{abstract}

Key words: HIV/AIDS, heterosexual, modelling, optimal control, condom.

\section{Introduction}

The extensive spread of human immuno-deficiency virus (HIV) and the associated acquired immuno-deficiency syndrome (AIDS) continues around the world. The pandemic has reached epidemic heights in most developing countries with the majority of cases in the sub-Saharan Africa. This has prompted different stake holders, organizations, scientists and political frontiers to assume one voice to combat and devise effective strategies to avert the destruction caused by the HIV/AIDS epidemic. Various chemotherapies of highly activity anti-retroviral therapy (HAART) drugs have been explored, efforts to find an effective and affordable preventive vaccine are on-going, laboratory research on HAART drugs has seen the introduction of a new family of drugs (fusion inhibitors (FIs)), campaigns to educate people about HIV/AIDS and on con- 
dom usage (on safe sex) are being intensified. However, irrespective of all these efforts total eradication of the HIV/AIDS epidemic is still elusive.

Up to date, there is no approved preventive vaccine for HIV/AIDS, therefore HAART drugs and condoms are the only available preventive control strategies. Most of HAART drugs fall into the following categories of either reverse transcriptase inhibitors (RTIs), protease inhibitors (PIs) and FIs. These drugs work by interfering with some aspects of the viral life cycle. RTIs inhibit HIV RNA from being converted into DNA, thus blocking integration of the viral code into the target cell [1]. On the other hand, PIs function by preventing the assembly of key viral proteins after they have been produced by the infected host cell. Therefore, PIs effectively reduce the number of infectious virus particles released by an infected cell. A third category of HIV drugs is a new family of anti-retrovirals presently represented only by one drug, enfuvirtide (T-20), called fusion/entry inhibitors [2, 16, 18]. Enfuvirtide is the first fusion inhibitor, but many other compounds are in the process of clinical development $[2,12,13,16]$. FIs work by inhibiting viral entry into CD4 $+\mathrm{T}$ cells $[2,12,13,16]$. Also, condoms can be used as a tool for controlling the spread of HIV. Clinical studies and experiments estimate that condom use is associated with $60-90 \%$ effectiveness, with a mean of $87 \%$ in reducing transmission [11]. A good example on the effectiveness of condom usage is evidenced from Thailand. The Government of Thailand implemented a policy, which required $100 \%$ condom usage by commercial sex workers and their clients. This led to an increase in the use of condoms from $14 \%$ in 1989 to $94 \%$ in 1994, and a decrease in sexually transmitted infections from 410,406 in 1987 to 29,362 in 1994 [14].

A number of research efforts with aid of mathematical models has been employed to assess the effects of vaccine, condom use and HAART drugs in the control of HIV at cell level and population level $[5,10,11,14,15,17]$. Also, several optimal control options have been proposed that were derived from dynamic with in-host HIV models $[1,4,7,8]$ and it still remains imperative to explore possible HIV intervention schemes based on HIV population dynamics. We also mention a paper by Tumwiine [19] where a stability analysis is done for a malaria model. Since, HAART drugs and condoms are the only intervention strategies that are currently available, we therefore focus in this study on devising an optimal scheme on the implementation of condom usage in a poor resource setting such as Zimbabwe to reduce HIV prevalence. Sub-Saharan countries like Botswana, South Africa and Zimbabwe have been introduced to HAART drugs and condoms in a bid to reduce the HIV prevalence in the region. However, availability of drugs and condoms in the remote areas of this region is a major concern. The cost of these intervention options is beyond reach of many infected patients, hence a need to come up with a scheme by their governments, health and donor organizations, and other interested parties to make drugs and condoms accessible at affordable prices to their people. Such a scheme should be followed by campaigns on educating people on condom usage, supply of condoms freely or at subsidized prices, travelling to remote areas deploying and training health workers. The main challenge with such a campaign is the cost associated with the activities that need to 
be carried out to ensure success of the scheme. We seek to derive an optimal control scheme that will minimise the cases of infectious HIV, minimise the cost of implementing condoms as an intervention strategy and maximise the population of health people.

This paper is organized as follows, in Section 2 we present a sex-structured HIV model suggested by Mukandavire and Garira [14]. In Section 2, we also incorporate the intervention of condoms and develop the objective functional to determine an optimal control strategy that involves condom usage in a population. Existence and characterization of the optimal control are carried out in Section 3 and 4, respectively. The optimality system is given in Section 5. In Section 6 we present the numerical simulations of the optimal system. Concluding remarks are provided in Section 7.

\section{$2 \mathrm{HIV} / \mathrm{AIDS}$ Model}

We begin by presenting a modified version of a sex-structured HIV/AIDS model proposed by Mukandavire and Garira [14]. The model, describes the interaction of susceptibles $S_{i}(t)$, containing individuals in sex group $i$, who are not infected with the virus, infectives $I_{i}(t)$, containing individuals who are infected with the virus but have not yet developed AIDS symptoms. AIDS cases $A_{i}(t)$, are those individuals in sex group $i$ who have developed fully symptomatic AIDS and exhibit specific clinical features, where $i=f, m$ denote female and male, respectively. In this model, it is assumed that new recruits enter the heterosexually active population at a rate $\Lambda$. A proportion $\rho$ of these individuals are assumed to be female susceptibles (categorized in the class $S_{f}$ ) and the complementary proportion $(1-\rho)$, are male susceptibles $\left(S_{m}\right)$. After acquiring infection female and male susceptibles enter the classes $I_{f}$ and $I_{m}$ of infected individuals, respectively. All the people in the different classes have a natural death rate of $\mu(>0)$. AIDS patients have an additional diseaseinduced mortality rate $v(>0)$, and there is constant exit of people at a rate $\alpha(>0)$, migrating to developed countries in pursuit of education and employment, except for AIDS patients. The force of infection of this model is given by $\lambda_{j}=\beta_{j} c_{i} I_{j}(t) / N_{j}(t)$, where $i, j=f, m, i \neq j$, and $N_{j}(t)=S_{j}(t)+I_{j}(t)$. The force of infection $\lambda_{j}$ is the average per capita risk of infection of individuals infected per unit time, where $\beta_{i}$ is the probability of transmission and $c_{i}$ is the rate at which individuals acquire new sexual partner per unit time.

Modelling Condom Use in HIV Dynamics. When condoms are introduced in a population as an intervention strategy the force of infection $\lambda_{j}$ will be modified to $\lambda_{j}^{c}=\beta_{j} c_{i}\left(1-u_{c}\right) I_{j}(t) / N_{j}(t)$, where $u_{c}$ represents the percentage of preventability of infection transmission when condoms are used. For simplicity, $u_{c}$ represents both male and female condom use in a community.

$$
\begin{aligned}
& S_{f}^{\prime}(t)=\rho \Lambda-\beta_{m} c_{f} \frac{\left(1-u_{c}\right) S_{f}(t) I_{m}(t)}{N_{m}(t)}-(\mu+\alpha) S_{f}(t), \\
& I_{f}^{\prime}(t)=\beta_{m} c_{f} \frac{\left(1-u_{c}\right) S_{f}(t) I_{m}(t)}{N_{m}(t)}-(\gamma+\mu+\alpha) I_{f}(t),
\end{aligned}
$$




$$
\begin{aligned}
& A_{f}^{\prime}(t)=\gamma I_{f}(t)-(\mu+v) A_{f}(t), \\
& S_{m}^{\prime}(t)=(1-\rho) \Lambda-\beta_{f} c_{m} \frac{\left(1-u_{c}\right) S_{m}(t) I_{f}(t)}{N_{f}(t)}-(\mu+\alpha) S_{m}(t), \\
& I_{m}^{\prime}(t)=\beta_{f} c_{m} \frac{\left(1-u_{c}\right) S_{m}(t) I_{f}(t)}{N_{f}(t)}-(\gamma+\mu+\alpha) I_{m}(t), \\
& A_{m}^{\prime}(t)=\gamma I_{m}(t)-(\mu+v) A_{m}(t) .
\end{aligned}
$$

This model does not consider the movement of infectives $\left(I_{f}\right.$ and $\left.I_{m}\right)$ to the AIDS classes $\left(A_{f}\right.$ and $\left.A_{m}\right)$ using a discrete time delay, respectively (as in the study [14]), but assumes a constant movement of infectives to the AIDS classes at rate $\gamma$.

We define our objective functional as

$$
J\left(u_{c}(t)\right)=\int_{0}^{t_{f}}\left[S_{f}(t)+S_{m}(t)-B_{1} I_{f}(t)-B_{2} I_{m}(t)-B_{3} u_{c}(t)^{2}\right] d t .
$$

The first two terms represent the benefit of the susceptible female and male populations, the parameters $B_{1}$ and $B_{2}$ represent the weight constants for infectious female and male populations and $B_{3}$ the weight of the control, respectively. Our goal is to maximise the population of susceptibles, both males and females, minimise the populations of infectious males and females, maximise condom usage, while minimizing the cost of implementing condom usage in a population. The value $u_{c}=1$ represents maximal condom usage $(100 \%)$ in a population and the maximal cost of education, implementation and campaigns on condom usage in a population is represented by $u_{c}(t)^{2}$. We therefore seek an optimal control $u_{c}^{*}$ such that

$$
\begin{aligned}
& J\left(u_{c}^{*}\right)=\max \left\{J\left(u_{c}\right) \mid u_{c} \in U\right\}, \\
& U=\left\{u_{c}(t) \mid u_{c}(t) \text { is measurable }: 0 \leq a \leq u_{c}(t) \leq b \leq 1, t \in\left[0, t_{f}\right]\right\} .
\end{aligned}
$$

The basis framework of this problem is to characterised the optimal control and prove the existence of the optimal control.

\section{Existence of an Optimal Control}

The existence of an optimal control is proved by a result from Fleming and Rishel [3]. The boundedness of solutions of system of equations (2.1)-(2.6) for a finite time interval is used to prove the existence of an optimal control.

To determine existence of an optimal control to our problem, we use a result from Fleming and Rishel [3] (Theorem 4.1 pp. 68-69), where the following properties must be satisfied.

1. The set of controls and corresponding state variables is nonempty.

2. The control set $U$ is convex and closed.

3. The right hand side of the state system is bounded by a linear function in the state and control. 
4. The integrand of the functional is concave on $U$ and is bounded above by $c_{2}-c_{1}\left|u_{c}\right|^{\kappa}$, where $c_{1}, c_{2}>0$ and $\kappa>1$.

An existence result in Lukes [9] (Theorem 9.2.1) for the system of equations (2.1)-(2.6) for bounded coefficients is used to give condition 1. The control set is closed and convex by definition. The right hand side of the state system (equations (2.1)-(2.6)) satisfies condition 3 since the state solutions are a priori bounded. The integrand in the objective functional, $S_{f}(t)+S_{m}(t)-B_{1} I_{f}(t)-$ $B_{2} I_{m}(t)-B_{3} u_{c}(t)^{2}$, is concave on $U$. Furthermore, $c_{1}, c_{2}>0$ and $\kappa>1$, hence

$$
S_{f}(t)+S_{m}(t)-B_{1} I_{f}(t)-B_{2} I_{m}(t)-B_{3} u_{c}(t)^{2} \leq c_{2}-c_{1}\left|u_{c}\right|^{\kappa},
$$

therefore the optimal control exists, since the states are bounded.

\section{Characterization}

Since there exist an optimal control for maximizing the functional (2.7) subject to equations (2.1)-(2.6), we use Pontryagin's Maximum Principle to derive the necessary conditions for this optimal control. The lagrangian is defined as,

$$
\begin{aligned}
L & =S_{f}(t)+S_{m}(t)-B_{1} I_{f}(t)-B_{2} I_{m}(t)-B_{3} u_{c}(t)^{2} \\
& +\lambda_{1}\left[\rho \Lambda-\beta_{m} c_{f} \frac{\left(1-u_{c}\right) S_{f}(t) I_{m}(t)}{N_{m}(t)}-(\mu+\alpha) S_{f}(t)\right] \\
& +\lambda_{2}\left[\beta_{m} c_{f} \frac{\left(1-u_{c}\right) S_{f}(t) I_{m}(t)}{N_{m}(t)}-(\gamma+\mu+\alpha) I_{f}(t)\right]+\lambda_{3}\left[\gamma I_{f}(t)-(\mu+v) A_{f}(t)\right] \\
& +\lambda_{4}\left[(1-\rho) \Lambda-\beta_{f} c_{m} \frac{\left(1-u_{c}\right) S_{m}(t) I_{f}(t)}{N_{f}(t)}-(\mu+\alpha) S_{m}(t)\right] \\
& +\lambda_{5}\left[\beta_{f} c_{m} \frac{\left(1-u_{c}\right) S_{m}(t) I_{f}(t)}{N_{f}(t)}-(\gamma+\mu+\alpha) I_{m}(t)\right] \\
& +\lambda_{6}\left[\gamma I_{m}(t)-(\mu+v) A_{m}(t)\right]+w_{1}(t)(b-\gamma(t))+w_{2}(t)(\gamma(t)-a),
\end{aligned}
$$

where $w_{1}(t) \geq 0, w_{2}(t) \geq 0$ are penalty multipliers satisfying $w_{1}(t)(b-\gamma(t))+$ $w_{2}(t)(\gamma(t)-a)=0$ at the optimal $u_{c}^{*}$.

Theorem 1. Given an optimal control $u_{c}^{*}$ and solutions of the corresponding state system (2.1)-(2.6), there exist adjoint variables $\lambda_{i}, i=1, \ldots, 5$ satisfying

$$
\begin{aligned}
& \frac{d \lambda_{1}}{d t}=-1+\left(1-u_{c}\right)\left(\beta_{m} c_{f} \frac{I_{m}}{N_{m}}\left(\lambda_{1}-\lambda_{2}\right)+\beta_{f} c_{m} \frac{S_{m} I_{f}}{\left(S_{f}+I_{f}\right)^{2}}\left(\lambda_{5}-\lambda_{4}\right)\right)+(\mu+\alpha) \lambda_{1}, \\
& \frac{d \lambda_{2}}{d t}=B_{1}+\beta_{f} c_{m}\left(1-u_{c}\right) \frac{S_{m} S_{f}}{\left(S_{f}+I_{f}\right)^{2}}\left(\lambda_{4}-\lambda_{5}\right)+\alpha\left(\lambda_{2}-\lambda_{3}\right)+\lambda_{2}(\mu+\alpha), \\
& \frac{d \lambda_{3}}{d t}=(\mu+v) \lambda_{3}, \quad \frac{d \lambda_{6}}{d t}=(\mu+v) \lambda_{6} \\
& \frac{d \lambda_{4}}{d t}=-1+\left(1-u_{c}\right)\left(\beta_{m} c_{f} \frac{S_{f} I_{m}}{\left(S_{m}+I_{m}\right)^{2}}\left(\lambda_{2}-\lambda_{1}\right)+\beta_{f} c_{m} \frac{I_{f}}{N_{f}}\left(\lambda_{4}-\lambda_{5}\right)\right)+(\mu+\alpha) \lambda_{4}, \\
& \frac{d \lambda_{5}}{d t}=B_{2}+\beta_{m} c_{f}\left(1-u_{c}\right) \frac{S_{m} S_{f}}{\left(S_{m}+I_{m}\right)^{2}}\left(\lambda_{1}-\lambda_{2}\right)+\alpha\left(\lambda_{5}-\lambda_{6}\right)+\lambda_{5}(\mu+\alpha),
\end{aligned}
$$


with transversality conditions $\lambda_{i}\left(t_{f}\right)=0$ for $i=1, \ldots 6$. To determine the interior maximum of the given lagrangian function, we take the partial derivate of $L$ with respect to $u_{c}^{*}$ and set it to zero. Thus,

$$
-2 B_{3} u_{c}(t)^{*}+\left(\lambda_{1}-\lambda_{2}\right) \beta_{m} c_{f} \frac{S_{f} I_{m}}{N_{m}}+\left(\lambda_{4}-\lambda_{5}\right) \beta_{f} c_{m} \frac{S_{m} I_{f}}{N_{f}}-w_{1}(t)+w_{2}(t)=0
$$

an we get $u_{c}(t)^{*}$ as

$$
u_{c}(t)^{*}=\left(\lambda_{1}-\lambda_{2}\right) \beta_{m} c_{f} \frac{S_{f} I_{m}}{2 B_{3} N_{m}}+\left(\lambda_{4}-\lambda_{5}\right) \beta_{f} c_{m} \frac{S_{m} I_{f}}{2 B_{3} N_{f}}-\frac{w_{1}(t)-w_{2}(t)}{2 B_{3}} .
$$

To determine an explicit expression for the control without $w_{1}$ and $w_{2}$, a standard optimality technique is utilized. The following three cases are considered to determine a specific characterization of the optimal control.

(i) On the set $\left\{t \mid a<u_{c}(t)^{*}<b\right\}, w_{1}=0, w_{2}=0$. Hence the optimal control is

$$
u_{c}(t)^{*}=\left(\lambda_{1}-\lambda_{2}\right) \beta_{m} c_{f} \frac{S_{f} I_{m}}{2 B_{3} N_{m}}+\left(\lambda_{4}-\lambda_{5}\right) \beta_{f} c_{m} \frac{S_{m} I_{f}}{2 B_{3} N_{f}} .
$$

(ii) On the set $\left\{t \mid a=u_{c}(t)\right\}, w_{1}=0$, hence

$$
u_{c}(t)^{*}=\left(\lambda_{1}-\lambda_{2}\right) \beta_{m} c_{f} \frac{S_{f} I_{m}}{2 B_{3} N_{m}}+\left(\lambda_{4}-\lambda_{5}\right) \beta_{f} c_{m} \frac{S_{m} I_{f}}{2 B_{3} N_{f}}+\frac{w_{2}(t)}{2 B_{3}}
$$

or

$$
\left(\lambda_{1}-\lambda_{2}\right) \beta_{m} c_{f} \frac{S_{f} I_{m}}{2 B_{3} N_{m}}+\left(\lambda_{4}-\lambda_{5}\right) \beta_{f} c_{m} \frac{S_{m} I_{f}}{2 B_{3} N_{f}} \leq a .
$$

(iii) On the set $\left\{t \mid b=u_{c}(t)\right\}, w_{2}=0$, hence

$$
u_{c}(t)^{*}=\left(\lambda_{1}-\lambda_{2}\right) \beta_{m} c_{f} \frac{S_{f} I_{m}}{2 B_{3} N_{m}}+\left(\lambda_{4}-\lambda_{5}\right) \beta_{f} c_{m} \frac{S_{m} I_{f}}{2 B_{3} N_{f}}-\frac{w_{1}(t)}{2 B_{3}}
$$

or

$$
\left(\lambda_{1}-\lambda_{2}\right) \beta_{m} c_{f} \frac{S_{f} I_{m}}{2 B_{3} N_{m}}+\left(\lambda_{4}-\lambda_{5}\right) \beta_{f} c_{m} \frac{S_{m} I_{f}}{2 B_{3} N_{f}} \geq b
$$

Combining these three cases, the optimal control is characterised as,

$$
u_{c}(t)^{*}=\min \left\{\max \left\{a,\left(\lambda_{1}-\lambda_{2}\right) \beta_{m} c_{f} \frac{S_{f} I_{m}}{2 B_{3} N_{m}}+\left(\lambda_{4}-\lambda_{5}\right) \beta_{f} c_{m} \frac{S_{m} I_{f}}{2 B_{3} N_{f}}\right\}, b\right\} .
$$




\section{The Optimal System}

The optimality system consists of the state system coupled with the adjoint system with the initial conditions, the transversality conditions and the characterization of the optimal control.

$$
\begin{aligned}
& S_{f}^{\prime}(t)=\rho \Lambda-\beta_{m} c_{f} \frac{\left(1-u_{c}^{*}\right) S_{f}(t) I_{m}(t)}{N_{m}(t)}-(\mu+\alpha) S_{f}(t), \\
& I_{f}^{\prime}(t)=\beta_{m} c_{f} \frac{\left(1-u_{c}^{*}\right) S_{f}(t) I_{m}(t)}{N_{m}(t)}-(\gamma+\mu+\alpha) I_{f}(t), \\
& A_{f}^{\prime}(t)=\gamma I_{f}(t)-(\mu+v) A_{f}(t), \\
& S_{m}^{\prime}(t)=(1-\rho) \Lambda-\beta_{f} c_{m} \frac{\left(1-u_{c}^{*}\right) S_{m}(t) I_{f}(t)}{N_{f}(t)}-(\mu+\alpha) S_{m}(t), \\
& I_{m}^{\prime}(t)=\beta_{f} c_{m} \frac{\left(1-u_{c}^{*}\right) S_{m}(t) I_{f}(t)}{N_{f}(t)}-(\gamma+\mu+\alpha) I_{m}(t), \\
& A_{m}^{\prime}(t)=\gamma I_{m}(t)-(\mu+v) A_{m}(t), \\
& \frac{d \lambda_{1}}{d t}=-1+\left(1-u_{c}^{*}\right)\left(\beta_{m} c_{f} \frac{I_{m}}{N_{m}}\left(\lambda_{1}-\lambda_{2}\right)+\beta_{f} c_{m} \frac{S_{m} I_{f}}{\left(S_{f}+I_{f}\right)^{2}}\left(\lambda_{5}-\lambda_{4}\right)\right)+(\mu+\alpha) \lambda_{1}, \\
& \frac{d \lambda_{2}}{d t}=B_{1}+\beta_{f} c_{m}\left(1-u_{c}^{*}\right) \frac{S_{m} S_{f}}{\left(S_{f}+I_{f}\right)^{2}}\left(\lambda_{4}-\lambda_{5}\right)+\alpha\left(\lambda_{2}-\lambda_{3}\right)+\lambda_{2}(\mu+\alpha), \\
& \frac{d \lambda_{3}}{d t}=(\mu+v) \lambda_{3}, \quad \frac{d \lambda_{6}}{d t}=(\mu+v) \lambda_{6}, \\
& \frac{d \lambda_{4}}{d t}=-1+\beta_{m} c_{f}\left(1-u_{c}^{*}\right) \frac{S_{f} I_{m}}{\left(S_{m}+I_{m}\right)^{2}}\left(\lambda_{2}-\lambda_{1}\right)+\beta_{f} c_{m}\left(1-u_{c}^{*}\right) \frac{I_{f}}{N_{f}}\left(\lambda_{4}-\lambda_{5}\right) \\
& \frac{d \lambda_{5}}{d t}=B_{2}+\beta_{m} c_{f}\left(1-u_{c}^{*}\right) \frac{S_{m} S_{f}}{\left(S_{m}+I_{m}\right)^{2}}\left(\lambda_{1}-\lambda_{2}\right)+\alpha\left(\lambda_{5}-\lambda_{6}\right)+\lambda_{5}(\mu+\alpha), \\
& +(\mu+\alpha) \lambda_{4},
\end{aligned}
$$

where $u_{c}(t)^{*}$ is given by expression (4.3), with $S_{m}(0)=S_{m_{0}}, S_{f}(0)=S_{f_{0}}$, $I_{m}(0)=I_{m_{0}}, I_{f}(0)=I_{f_{0}}, A_{m}(0)=A_{m_{0}}, A_{f}(0)=A_{f_{0}}$ and $\lambda_{i}\left(t_{f}\right)=0$ for $i=1, \ldots, 6$.

Due to a priori boundedness of the state and adjoint functions and the resulting Lipschitz structure of the ODEs, we obtain the uniqueness of the optimal control for small $t_{f}[6]$. The uniqueness of the optimal control follows from the uniqueness of the optimality system.

\section{Numerical Simulations}

The optimality system is solved by using an iterative method with RungeKutta fourth order method. Starting with a guess for the adjoint variables, the state equations are solved forward in time. Then those state values are used to solve the adjoint equations backward in time, and the iterations continue until convergence. We carry simulations to determine how maximizing percentage of condom use enhances HIV prevalence. The effects of costs that can be incurred 
Table 1. Data for the sex-structured HIV/AIDS model.

\begin{tabular}{lll}
\hline Parameter & Value & Source \\
\hline Birth rate $(\Lambda)$ & 0.029 year $^{-1}$ & Central Statistic Office \\
Proportion of mature female $(\rho)$ & 0.5 & Central Statistic Office \\
Adult death rate $(\mu)$ & 0.02 year $^{-1}$ & Central Statistic Office \\
AIDS related death rate $(v)$ & $0.333^{-1}$ & Central Statistic Office \\
Emigration rate $(\alpha)$ & 0.01 & Central Statistic Office \\
Rate of acquiring sexual partners $\left(c_{f}, c_{m}\right)$ & 3.0 & {$[14]$} \\
Transmission probability $\left(\beta_{f}, \beta_{m}\right)$ & $0.097,0.1637$ & {$[14]$} \\
Progression to AIDS $(\gamma)$ & 0.045 & Estimated \\
\hline
\end{tabular}

in implementing condoms as an intervention that include campaigns, television advertisements, education on safe usage and acceptability of use by both males and females are also studied to evaluate how these costs can affect the control of HIV transmission. We increased the value of $B_{3}$ (the cost weight) in Figure 1 to asses how the population of susceptibles, infectives and AIDS cases are altered. In Figure 2, we investigate the impact of increasing minimization of infectives through increasing the weights $B_{1}$ and $B_{2}$ impact the control of HIV transmission. These simulations are carried out with initial values of the state variables given as $S_{m}(0)=11000000.0, S_{f}(0)=11000000.0, I_{m}(0)=$ $500000.0, I_{f}(0)=500000.0, A_{m}(0)=500000.0$, and $A_{f}(0)=500000.0$. Figure 1 shows that as the costs of the condom campaign, that includes education, supply of free and cheap condoms, and training health workers increase, the percentage of condom usage will drop early (the campaign will be withdrawn early). That is the more people are educated on condom use and can easily access them cheaply or get them freely, the condom percentage remains high.

But as education campaigns become expensive, condoms un-accessible and un-affordable their usage drops and cases of infectious HIV increases. Figures 1 (d), (e), (f) and (g) show that with condom usage the population of infectious male and female decrease and number of AIDS cases drop with high percentage usage of condoms. However, if percentage usage decreases the rate of reduction of AIDS cases and populations of infectious individuals is altered. Figures 1 (a) and (b) show that condom usage reduces transmission of infection to the susceptibles, hence an increase in the population of the susceptibles. Figures 2 (a) and (b) show that increasing the weights $B_{1}$ and $B_{2}$ (increasing $B_{1}$ and $B_{2}$ implies increasing the minimization of $I_{m}$ and $I_{f}$ ) enables pro-longed issuing of cheap condoms or implementation of the campaign for a longer time. The objective functional seeks to minimise the cases of infectious HIV, which could be achieved by reducing transmission of infection to the susceptibles by the infectives through condom usage. Since campaigns costs work against achieving the goals of the scheme, then theoretically issuing condoms freely to those that are infectious will cut costs (this enhance reduced transmission of infection from $I_{m}$ and $I_{f}$ to the susceptibles) than making condoms available to everyone even those without the potential to transmit the infection (for example the susceptibles which are the low risk group). Such an implementation is similar to the policy of percentage condom usage by commercial sex workers (high risk group) that was implemented by the the government of Thailand. However, 
Optimal Control of a Sex-Structured HIV/AIDS Model with Condom Use 491

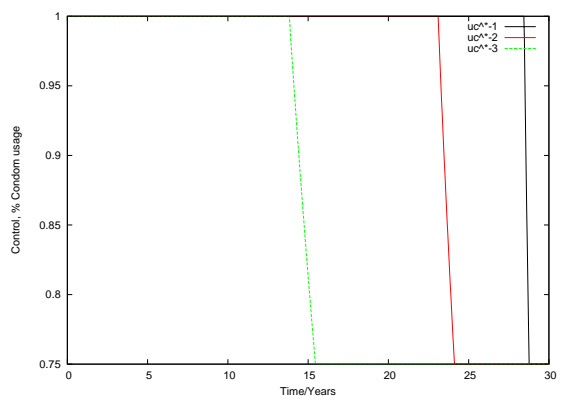

a)

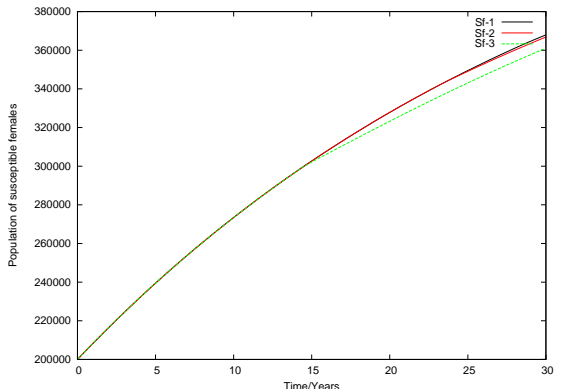

c)

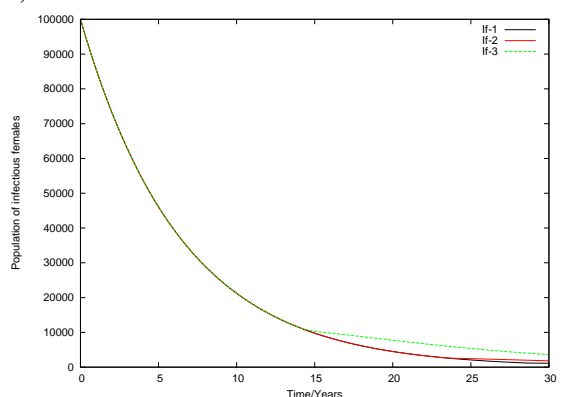

e)

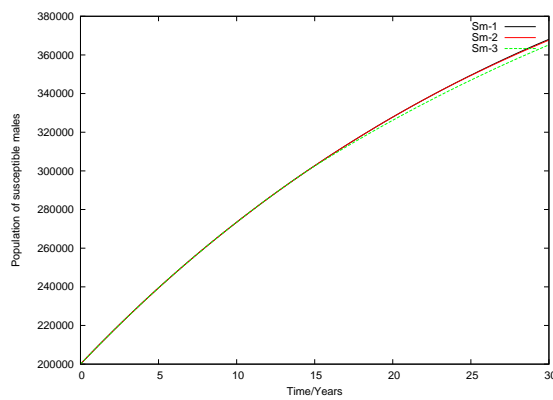

b)

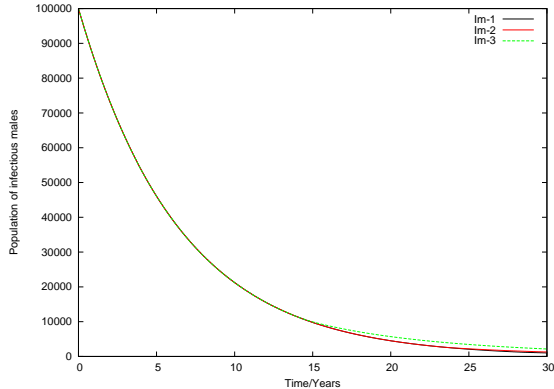

d)

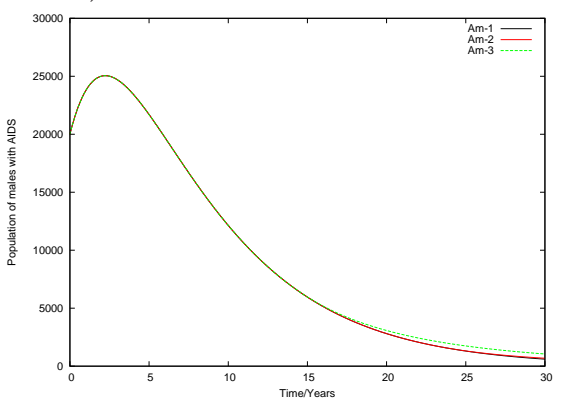

f)

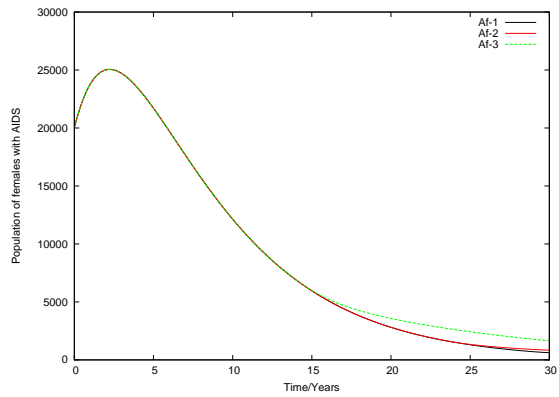

e)

f)

Figure 1. Graphs of the numerical solutions of the optimality system, showing propagation of (a) \% condom usage, (b) susceptible males, (c) susceptible females, (d) infectious males, (e) infectious females, (f) male AIDS cases, and (g) female AIDS cases when condoms are used as an intervention for 30 years. The value of the weights used are (i) $B 3=500$ corresponds to variables with subscript 1 , (ii) $B 3=50000$ corresponds to variables with subscript 2 , and (iii) $B 3=50$ corresponds to variables with subscript 3 .

Math. Model. Anal., 14(4):483-494, 2009. 


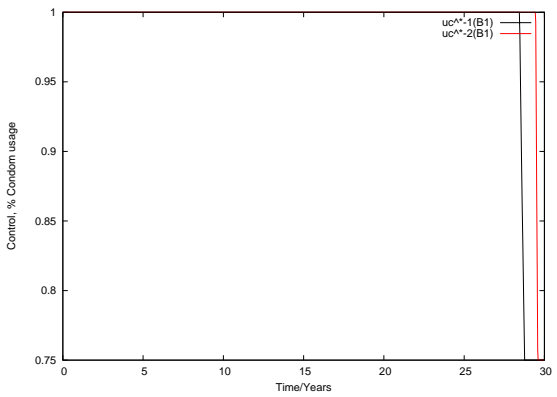

a)

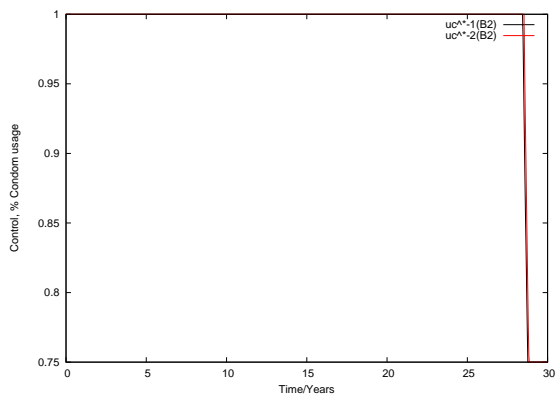

b)

Figure 2. Graphs of the numerical solutions to the behaviour of the control constrain $u_{c}^{*}$, (a) when $B 1(1)=0.025$ and $B 1(2)=2.5$, and (b) when $B 2(1)=0.025$ and $B 2(2)=25.0$. With initial conditions similar to conditions in Figure 1.

such a practice is difficult since it requires diagnosis before issuing of condoms. One of the biggest stabling block in the campaign of condom usage in the African setting is the acceptance of the female condom and the associated stigma with their counterparts for women suggesting to use a female condom or insisting that the male counterpart should use a condom. This is where the educational campaigns are supposed to break the African barriers and ensure acceptance of condom use by both males and females.

\section{Discussion}

This study suggests that very high percentage of condom use can help to reduce the prevalence of HIV in the sub-Saharan region. However, the challenge is the cost that people in this region are unable to meet that paralysis their potential (condoms) to effectively reduce the HIV prevalence. If condoms could be made easily available at affordable prices in all remote areas of the region then more positive results can be achieved. Nevertheless, if condom availability is reduced, their cost become unbearable, if the acceptance of their use is still questionable, and people are still ignorant concerning their use through failure of the condom usage implementation campaigns; then condom usage as an intervention strategy will not achieve the desired result. Increases in the costs of the condom implementation campaigns reduce the percentage of condoms used in the population that leads to less reduction of AIDS and infectious HIV cases. A possible way to reduce campaign costs, is to target issuing of condoms to those in the infectious stage (the high risk group) and ensure $100 \%$ usage by people in such categories than making condoms freely available to people who do not make any threat of transmitting the infection; for example, the policy that was implemented by the government of Thailand that enforced $100 \%$ condom use by commercial sex (the high risk group) workers. This achieves minimization of the infection transmission by both infectious males and females and cut costs of cheap or free condom supply and ensure high percentage use of condoms by those who are highly infectious. Control programs that follow such 
control protocols can effectively reduce the number of infectious and HIV/AIDS cases in the sub-Saharan Africa.

\section{Acknowledgments}

The authors would like to thank Professor S. Lenhart for optimal control literature she supplied.

\section{References}

[1] B.M. Adams and et al. Dynamic multidrug therapies for HIV: optimal and STI control approaches. J M.B.E., 1(2):223-241, 2004.

[2] V. Briz, E. Poveda and V. Soriano. HIV entry inhibitors: mechanisms of action and resistance pathways. J. Antimic. Chemo., 57:619-627, 2006. (doi:10.1093/jac/dkl027)

[3] W.H. Fleming and R.W. Rishel. Deterministic and Stochastic Optimal Control. Spinger, New York, 1975.

[4] W. Garira, S.D. Musekwa and T. Shiri. Optimal control of combined therapy in a single strain HIV-1 model. EJDE, 52:1-22, 2005.

[5] D. Greenhalgh, M. Dolye and F. Lewis. A mathematical treatment of AIDS and condom use. IMA J. Math. Appl. Med. Biol., 18(3):225-262, 2001. (doi:10.1093/imammb/18.3.225)

[6] H.R. Joshi. Optimal control of an HIV immunology model. Opt. Control Appl. Meth., 23:199-213, 2002. (doi:10.1002/oca.710)

[7] D. Kirschner and G.F. Wedd. A mathematical model of combined drug therapy of HIV infection. J. Theor. Med.1, pp. 25-34, 1997.

[8] H. Kwon. Optimal treatment strategies derived from a HIV model with drug resistant mutants. A. Math. Comp., 188(2):1193-1204, 2007. (doi:10.1016/j.amc.2006.10.071)

[9] D.L. Lukes. Differential Equations: Classical to controlled. Mathematical in Science and Engineering. Academic Press, New York, 1982.

[10] G. Magombedze, W. Garira and E. Mwenje. Modelling the immuno-pathogenesis of HIV-1 infection and the effect of mlti-drug therapy: The role of Fusion inhibitors in HAART. Math. Biosc. Eng., 5(3):485-504, 2008.

[11] S.M. Moghadas, A.B. Gumel, R.G. McLeod and R. Gordon. Could condoms stop the AIDS epidemic. J. Theor. Med., 5:172-181, 2003.

[12] J.P. Moore and R.W. Doms. The entry of entry inhibitors: A fusion of science and medicine. PNAS, 100(19):10598-10602, 2003. (doi:10.1073/pnas.1932511100)

[13] G. Moyle. Stopping HIV fusion with enfuvirtide: the first step to extracellular HAART. J. Antimic. Chemo., 51:213-217, 2003. (doi:10.1093/jac/dkg066)

[14] Z. Mukandavire and W. Garira. Sex-structured HIV/AIDS model to analyse the effects of condom use with application to Zimbabwe. J. Math. Biol., 54:669-699, 2007. (doi:10.1007/s00285-006-0063-5)

[15] Z. Mukandavire and Garira W. HIV/AIDS model for assessing the effects of prophylactic sterilizing vaccines, condoms and treatment with amelioration. Bio. Sys., 14(3):323-355, 2006. 
[16] J.K. Rockstroh and S. Mauss. Clinical perspective of fusion inhibitors for treatment of HIV. J. Antimic. Chemo., 53:700-702, 2004. (doi:10.1093/jac/dkh161)

[17] T. Shiri, W. Garira and S.D. Musekwa. A two-strain HIV-1 mathematical model to assess the effects of chemotherapy on disease parameters. MBE Journal, 2:811-832, 2005 .

[18] T.K. Tashima and et al. Fusion inhibition a major but costly step forward in the treatment of HIV-1. N. ENGL. J. MED., 348(22):2249-2250, 2003. (doi:10.1056/NEJMe030042)

[19] J. Tumwiine, J.Y.T. Mugisha and L.S. Luboobi. Threshold and stability results for a malaria model in a population with protective intervention among high-risk groups. Math. Model. Anal., 13(3):443-460, 2008. (doi:10.3846/1392-6292.2008.13.443-460) 